\title{
Exciting Dressed BICs Via Photon Scattering and Delayed Quantum Feedback ${ }^{\dagger}$
}

\author{
Giuseppe Calajó ${ }^{1,2}$, Yao-Lung L. Fang ${ }^{3,4,5}$, Harold U. Baranger ${ }^{6}$ and Francesco Ciccarello $7,8, *$ (D) \\ 1 ICFO-Institut de Ciencies Fotoniques, The Barcelona Institute of Science and Technology, 08860 Castelldefels \\ (Barcelona), Spain; giuseppe.calajo@icfo.eu \\ 2 Vienna Center for Quantum Science and Technology, Atominstitut, TU Wien, Stadionallee 2, \\ 1020 Vienna, Austria \\ 3 Department of Physics, Duke University, P.O. Box 90305, Durham, NC 27708-0305, USA; leofang@bnl.gov \\ 4 Computational Science Initiative, Brookhaven National Laboratory, Upton, NY 11973, USA; \\ 5 National Synchrotron Light Source II, Brookhaven National Laboratory, Upton, NY 11973, USA \\ 6 NEST, Istituto Nanoscienze-CNR, 41125 Modena, Italy; francesco.ciccarello@unipa.it \\ 7 Università degli Studi di Palermo, Dipartimento di Fisica e Chimica-Emilio Segrè, via Archirafi 36, \\ I-90123 Palermo, Italy \\ * Correspondence: francesco.ciccarello@unipa.it \\ + Presented at the 11th Italian Quantum Information Science conference (IQIS 2018), Catania, Italy, \\ 17-20 September 2018.
}

Published: 2 July 2019

\begin{abstract}
We consider a semi-infinite waveguide with linear dispersion coupled to a qubit, in which a dressed bound state in the continuum (BIC) is known to exist. We predict that this BIC can be excited with significant probability via multi-photon scattering in the non-Markovian regime where the photon delay time (corresponding to the qubit-mirror distance) is of the order of the qubit's decay time. A similar process excites the BIC existing in an infinite waveguide coupled to a pair of qubits, yielding stationary entanglement between them. This shows, in particular, that photon trapping via scattering can occur without band-edge effects or cavities, the essential resource being instead the delayed quantum feedback due to the mirror.
\end{abstract}

Keywords: waveguide QED; non-Markovian open quantum systems; delayed quantum feedback; quantum optics

\section{Introduction}

Waveguide Quantum ElectroDynamics (QED) [1,2] is a new research area [3] investigating the coherent interaction between quantum emitters and the one-dimensional (1D) field of a waveguide. As a major motivation prompting research on this topic, waveguide-QED setups are potentially an ideal platform to carry out quantum information processing tasks, especially quantum communication [4]. An intriguing effect arising from the 1D nature of the field in such setups is the formation of a dressed bound in the continuum state (BIC) [5]. This BIC can exist even with a single qubit in a semi-infinite waveguide [6,7] causing, in particular, incomplete decay or even full suppression of decay as experimentally observed in a circuit-QED waveguide [8]. The field component of such BIC consists of a single photon strictly confined in the interspace between the qubit and the waveguide's end (the latter embodies an effective mirror). This feature, alongside the fact that-by definition-a BIC does not decay, enable potential applications such as quantum memories where light trapping at the few-photon level is demanded [4,9].

A natural way to populate the BIC is to excite the qubit and let it decay in vacuum, a method that however becomes ineffective at large time delays $\tau$ in which case the BIC's photon component 
is dominant [6] ( $\tau$ is the time taken by a photon to complete a round-trip between the qubit and mirror). Such non-Markovian regime of non-negligible delays thus requires exciting the BIC's photonic component, a task typically accomplished through photon scattering. Single-photon scattering, yet, is unable to excite the BIC since it involves only unbound dressed states. Population of a bound state is however potentially achievable via multi-photon scattering, as shown by Longo et al. [10] for two-photon wavepackets and the normal bound state existing in the band gap of an array of resonators coupled to a qubit $[11,12]$. This possibility is due to the qubit's intrinsic nonlinearity as illustrated next. In single-photon scattering, the dynamics occurs in the single-excitation sector of the Hilbert space (assuming the rotating wave approximation (RWA)). In such a case, the qubit behaves exactly as a bosonic mode. This is no longer true if two photons are injected, yet one can still treat the qubit as a bosonic mode of equal frequency side-coupled to the waveguide provided that a fictitious on-site photon-photon repulsion term is added to the Hamiltonian (see e.g., [13]). Such repulsive interaction in particular mixes fully unbound stationary states (two photons scattering off the qubit) with semi-bound ones that correspond instead to one scattering photon plus one photon bound in the BIC (this mixing would not occur if the emitter were a true bosonic mode and thus the dynamics fully linear). Thereby, the scattering process where two incoming photons result in the BIC and only one fully scattered photon is generally possible.

\section{Results}

We theoretically show [14] that the aforementioned dressed BIC existing in a semi-infinite waveguide coupled to one qubit can be excited via multi-photon scattering with significant probability. Specifically, the setup considered is a waveguide with linear dispersion coupled under the RWA to a qubit placed at a distance $a$ from the mirror. Let $k_{0}$ be the wavevector of a photon resonant with the qubit, $\Gamma$ the qubit'decay rate into the waveguide (without mirror) and $\tau=2 a / v$ the delay time with $v$ the photon velocity. We find that a two-photon wavepacket of bandwidth $\Delta k$ sent on the qubit (towards the mirror) can happen to not be fully reflected back: with probability $P_{\mathrm{BIC}}$, only one photon is scattered off while another photon remains trapped in the qubit-mirror interspace populating the BIC. This effect occurs (namely $P_{\mathrm{BIC}}$ is significant) when the following three conditions are matched at once:
(i) $k a=m \pi$,
(ii) $\Delta k \sim \Gamma / v$,
(iii) $\Gamma \tau \sim 1$ or larger,

where $m=0,1, \ldots$. Condition (i) ensures the existence of the BIC [6]. Condition (ii), meaning that the wavepacket must have a finite bandwidth of the order of the decay rate, agrees with general expectations [15]. Condition (iii) is the most significant one: it states that the time delay must be long enough in order for BIC generation to be successful. This is in striking contrast with the approach of generating the BIC via qubit decay (see Introduction), which works best in the Markovian regime of negligible delays, $\Gamma \tau \ll 1$. Exciting the BIC via multi-photon scattering can therefore be regarded, in particular, as a non-Markovian effect: a finite memory is essential for its working principle.

These conclusions were assessed with different incoming pulses: two photons of equal frequency, two photons detuned oppositely in energy and a low-power coherent-state pulse [14]. In particular, coherent states of average photon number $\langle n\rangle \simeq 2$ yield values of $P_{\mathrm{BIC}}$ comparable with a pure two-photon wavepacket of the same shape, which bodes well for an experimental implementation. While most of these outcomes were obtained by considering for simplicity pulses with exponential shape, we gathered numerical evidence that wavepacket engineering can strongly enhance $P_{\mathrm{BIC}}$ (in a paradigmatic instance we found $P_{\mathrm{BIC}} \sim 80 \%$ [14]).

We note that, during the preparation of the present work, we became aware of a related scheme by Cotrufo and Alù [16] to populate the BIC arising for an atom-cavity system side-coupled to an infinite waveguide. Such a BIC however has no component over the waveguide field (unlike the BIC we focus on [6]). Additionally, Ref. [16] makes use of an emitter and a pair of cavities, while the present scheme relies solely on the feedback provided by the waveguide end. 
A BIC very similar in nature to the one discussed thus far occurs for an infinite waveguide (no mirror) coupled to two qubits placed at distance $a$ from each other [17,18]. Interestingly, this BIC now features entanglement between the qubits (unless the time delay is very long). Thereby, exciting the BIC-which is carried out through photon scattering analogously to one qubit-now produces, in addition to photon trapping, stationary entanglement [14].

\section{Methods}

Our results were obtained numerically through two different methods. One follows Refs. $[15,19]$ where the Schrödinger equation is used to derive a closed $1+1$ dimensional delay partial differential equation for the probability amplitude that a photon lies at $x$ with the qubit excited, which is then solved through a tailored finite-difference time-domain (FDTD) code with multithreading support [20]. The second approach instead discretizes the waveguide turning it into a fictitious coupled-cavity array (tight-binding model) whose normal frequencies form a finite band. If the number of cavities $N$ is large enough (typically $N \sim 100$ ) and only photon frequencies close to the band center are considered, then the CCA is a very good approximation of the waveguide since in the vicinity of the the band center the dispersion law is linear.

Acknowledgments: We thank Peter Rabl, Shanhui Fan, and Tommaso Tufarelli for useful discussions. We acknowledge support from U.S.DOE, Division of Materials Sciences and Engineering under Grant No. DE-SC0005237, the Brookhaven National Laboratory's Laboratory Directed Research and Development project No. 17-029, and the Fulbright Research Scholar Program. G. C. acknowledges support from the Austrian Science Fund (FWF) through SFB FOQUS F40, DK CoQuS W 1210, and the START grant Y 591-N16. Y. -L. L. F. acknowledges support from BNL LDRD projects No. 17-029 and No. 19-002, and New York State Urban Development Corporation, d/b/a Empire State Devel- opment, under contract No. AA289. Part of the computation was completed using resources of the Center for Functional Nanomaterials, which is a U.S. DOE Office of Science Facility, and the Scientific Data and Computing Center, a component of the Computational Science Initiative, at Brookhaven National Laboratory under Contract No. DE-SC0012704.

\section{References}

1. Liao, Z.; Zeng, X.; Nha, H.; Zubairy, M.S. Photon transport in a one- dimensional nanophotonic waveguide QED system. Phys. Scr. 2016, 91, 063004.

2. Roy, D.; Wilson, C.M.; Firstenberg, O. Colloquium: Strongly interacting photons in one- dimensional continuum. Rev. Mod. Phys. 2017, 89, 021001.

3. The First Workshop on Waveguide QED Was Held in Mazara del Vallo, Italy, 4-8 June 2018. Available online: https: / wqed18.weebly.com (accessed on 28 June 2019)

4. Kimble, H.J. The quantum internet. Nature 2008, 453, 1023.

5. Hsu, C.W.; Zhen, B.; Stone, A.D.; Joannopoulos, J.D.; Soljacic, M. Bound states in the continuum. Nat. Rev. Mater. 2016, 1, 16048.

6. Tufarelli, T.; Ciccarello, F.; Kim, M.S. Dynamics of spontaneous emission in a single-end photonic waveguide. Phys. Rev. A 2013, 87, 013820.

7. Tufarelli, T.; Kim, M.S.; Ciccarello, F. Non-Markovianity of a quantum emitter in front of a mirror. Phys. Rev. A 2014, 90, 012113.

8. Hoi, I.-C.; Kockum, A.F.; Tornberg, L.; Pourkabirian, A.; Johansson, G.; Delsing, P.; Wilson, C.M. Probing the quantum vacuum with an artificial atom in front of a mirror. Nat. Phys. 2015, 11, 1045-1049.

9. Lvovsky, A.I.; Sanders, B.C.; Tittel, W. Optical quantum memory. Nat. Photonics 2009, 3, 706.

10. Longo, P.; Schmitteckert, P.; Busch, K. Few-photon transport in low-dimensional systems: Interaction-induced radiation trapping. Phys. Rev. Lett. 2010, 104, 023602.

11. Lombardo, F.; Ciccarello, F.; Palma, G.M. Photon localization versus population trapping in a coupled-cavity array. Phys. Rev. A 2014, 89, 053826.

12. Calajó, G.; Ciccarello, F.; Chang, D.; Rabl, P. Atom-field dressed states in slow-light waveguide QED. Phys. Rev. A 2016, 93, 033833.

13. Fang, Y.-L.L.; Zheng, H.; Baranger, H.U. One-dimensional waveguide coupled to multiple qubits: photon-photon correlations. EPJ Quantum Technol. 2014, 1, 3. 
14. Calajó, G.; Fang, Y.-L.L.; Baranger, H.U.; Ciccarello, F. Exciting a Bound State in the Continuum through Multi-Photon Scattering plus Delayed Quantum Feedback. Phys. Rev. Lett. 2019, 122, 073601.

15. Fang, Y.-L.L.; Ciccarello, F.; Baranger, H.U. Non-Markovian dynamics of a qubit due to single-photon scattering in a waveguide. New J. Phys. 2018, 20, 043035.

16. Cotrufo, M.; Alù, A. Single-photon embedded eigenstates in coupled cavity-atom systems. Optica 2019, 6, 799.

17. Gonzalez-Ballestero, C.; Garcia-Vidal, F.J.; Moreno, E. Non-Markovian effects in waveguide-mediated entanglement. New J. Phys. 2013, 15, 073015.

18. Facchi, P.; Kim, M.S.; Pascazio, S.; Pepe, F.V.; Pomarico, D.; Tufarelli, T. Bound states and entanglement generation in waveguide quantum electrodynamics. Phys. Rev. A 2016, 94, 043839.

19. Fang, Y.-L.L. FDTD: Solving 1+1D delay PDE in parallel. Comput. Phys. Commun. 2018, 235, 422-432.

20. This FDTD code is open-sourced at https://github.com/leofang/FDTD.

(C) 2019 by the authors. Licensee MDPI, Basel, Switzerland. This article is an open access article distributed under the terms and conditions of the Creative Commons Attribution (CC BY) license (http://creativecommons.org/licenses/by/4.0/). 